Łukasz Kaźmierski ${ }^{1}$, Anna Bajek${ }^{1}$, Robert Debski², Maciej Gagat ${ }^{3}$, Alina Grzanka ${ }^{3}$, Magdalena Bodnar ${ }^{4}$, Krzysztof Roszkowski ${ }^{5}$

${ }^{1}$ Department of Tissue Engineering, Collegium Medicum Bydgoszcz, Nicolaus Copernicus University, Poland

2Department of Pediatric Hematology and Oncology, Collegium Medicum Bydgoszcz, Nicolaus Copernicus University, Poland

${ }^{3}$ Department of Histology and Embryology, Collegium Medicum Bydgoszcz, Nicolaus Copernicus University, Poland

${ }^{4}$ Department of Clinical Pathomorphology, Collegium Medicum Bydgoszcz, Nicolaus Copernicus University, Poland

5 5epartment of Oncology, Radiotherapy and Gynecologic Oncology, Collegium Medicum Bydgoszcz, Nicolaus Copernicus University, Poland

\title{
Can bone marrow mesenchymal stem cells regenerate the myocardium?
}

\author{
Corresponding author: \\ Krzysztof Roszkowski, Department \\ of Oncology, Radiotherapy \\ and Gynecologic Oncology, \\ Collegium Medicum Bydgoszcz, \\ Nicolaus Copernicus University, \\ Poland \\ e-mail: roszkowskik@cm.umk.pl
}

Medical Research Journal 2018; Volume 3, Number 3, 153-158 10.5603/MRJ.2018.a0026 Copyright (C) 2018 Via Medica ISSN 2451-2591

\begin{abstract}
Background: Cardiovascular diseases are the serious clinical problem, especially the loss of viable myocytes. A new approach, which provides a novel method for the treatment, is a tissue engineering and regenerative medicine. One of the current cell types used as a source to improve cardiac tissue repair, are MSCs. The aim of this study was to check if the 5'azacitidine and growth factors from cardiomyocyte cell line initiate the differentiation of MSCs toward cardiomyocytes.

Material and methods: Bone marrow MSCs were isolated and their biological features have been characterized. Conditioned media were prepared with the use of 5'azacitidine and growth factors released from cardiomyocytes.

Results: The transdifferentiating process has been confirmed by the expression of specific muscle markers. Conditioned medium from cardiomyocyte cell line, as well as, 5'azacitidine induced muscle differentiation process in bone marrow MSCs in comparison to the control.

Conclusions: Presented in this study data support the conclusion that this concept may represent a promising strategy for the repair of cardiac tissue, however, further experiments are necessary.

Key words: cell therapy, cardiovascular disease, mesenchymal stem cells, tissue engineering
\end{abstract}

Med Res J 2018; 3 (3): 153-158

\section{Introduction}

Cardiovascular diseases are the leading cause of death worldwide [1]. Despite improvements in survival rates following myocardial infarction, reduction in cardiac function attributable to the irreversible loss of viable myocytes remains a major clinical problem [2]. Unfortunately, the only therapy available to reverse the decline in cardiac function is heart transplantation. However, this option is available to very few patients due to a shortage of donor hearts [3]. Newly emerging technology, which may provide a novel method for the treatment of cardiovascular diseases, is regenerative medicine. Stem cell transplantation is a potential approach to repopulate injured myocardium, to treat heart failure and restore cardiac function [1]. A variety of cell types have been examined for their potential to achieve this type of cardiac reconstitution, but few have been shown to differentiate into myocytes capable of connecting with residual cardiomyocytes both mechanically and electrically following implantation [4]. Bone marrow Mesenchymal Stem Cells (MSCs) are one of the current cell types being studied as a source of factors to improve tissue repair [5]. Autologous MSCs are capable of differentiating into adipogenic, chondrogenic, osteogenic, and myogenic cell lines $[1,6]$. The relatively wide range of differentiation, coupled with high proliferation rate and relative ease of isolation, has made these cells suitable candidates for heart failure autologous cell therapy applications. The success of such therapy depends on the ability of stem cells transdifferentiation into cardiomyocytes in vitro and in vivo.

The aim of this study was to create conditions mimicking cardiomyoblasts environment. We have checked how 5 'azacitydyne and growth factors from cardiomyocyte cell line influenced on transdifferentiation of bone marrow mesenchymal stem cells into cardiomyocytes phenotype. 


\section{Materials and methods}

\section{Isolation and culture of bone marrow mesenchymal stem cells}

Rat bone marrow mesenchymal stem cells were isolated according to a protocol modified from Lennon and Caplan [7]. Briefly, bone marrow was collected by flushing femurs with complete medium. Cells were then washed and centrifuged twice in PBS (PAA, Austria), plated in Petri dishes and cultivated in $37^{\circ} \mathrm{C}$. After 3 days, nonadherent cells were removed and adherent cells were further cultured in complete medium for 14 days.

Basic culture medium for rat bone marrow mesenchymal stem cells was prepared containing DMEM/Ham's F12 (PAA, Austria) supplemented with 10\% FBS (PAA, Austria), $10 \mathrm{ng} / \mathrm{ml}$ bFGF (Sigma, Germany), $5 \mu \mathrm{g} / \mathrm{ml}$ amphotericin B (PAA, Austria) and $100 \mu \mathrm{g} / \mathrm{ml}$ penicillin/streptomycin (PAA, Austria).

The phenotype of mesenchymal stem cells was confirmed by colony forming efficiency assay and adipogenic and osteogenic assay. Briefly, for single cell approaches, equal numbers of isolated mesenchymal stem cells $\left(5 \times 10^{3}\right.$ cells $\left./ \mathrm{cm}^{2}\right)$ were plated in six-well culture plates. After 14 days of culture stem cell, colonies were visualized by staining with $2 \%$ rhodamine $B$ (Sigma, Germany). The colony forming efficiency (CFE) was calculated using the following formula: numbers of colonies formed/number of cells plated $\times 100 \%$.

Adipogenic differentiation was induced by adding the following reagents to the cell medium: DMEM/Ham's F12 (3:1) containing 10\% FBS, $10 \mu \mathrm{M}$ insulin (Sigma, Germany), $1 \mu \mathrm{M}$ dexamethasone (Sigma, Germany), $5 \mu \mathrm{g} / \mathrm{ml}$ amphotericin B and $100 \mu \mathrm{g} / \mathrm{ml}$ penicillin/streptomycin. Osteogenic differentiation was induced by the use of DMEM/Ham's F12 (3:1) medium containing 15\% FBS, $50 \mu \mathrm{M}$ ascorbic acid (Sigma, Germany), $0.1 \mu \mathrm{M}$ dexamethasone, $5 \mu \mathrm{g} / \mathrm{ml}$ amphotericin B and $100 \mu \mathrm{g} / \mathrm{ml}$ penicillin/streptomycin. Cells in both media were cultivated at $37^{\circ} \mathrm{C}$ in a humidified atmosphere with $5 \% \mathrm{CO}_{2}$ for 16 days.

\section{The culture of immortalized cardiomyocyte cell line}

Culture medium for immortalized cardiomyocyte cell line H9C2 (ATCC, USA) contained DMEM/Ham's F12, supplemented with $10 \%$ FBS, $5 \mu \mathrm{g} / \mathrm{ml}$ amphotericin B and $100 \mu \mathrm{g} / \mathrm{ml}$ penicillin/streptomycin. The harvested medium was designated conditioned media, filtered and stored at $-80^{\circ} \mathrm{C}$ until use.

Flow cytometry analysis of bone marrow mesenchymal stem cells

CD90 (10 $\mu /$ test, Chemicon, UK) and CD34 ( $1 \mu \mathrm{g} / 1 \times 10^{6}$ cell, Santa Cruz, USA) were used to characterize the primary cultures by flow cytometry. Cells were resuspended at a density of $1 \times 10^{6} \mathrm{cells} / \mathrm{ml}$ in cold PBS containing antibodies connected with FITC or phycoerythrin (Sigma, Germany). Labelling reaction was incubated in the dark for $30 \mathrm{~min}$. at $4^{\circ} \mathrm{C}$. After cells were washed three times and centrifuged, flow cytometry analysis was performed on a EPICS $\mathrm{XL}$ flow cytometry using System 2 Software Version 3.0 (Beckman-Coulter, Miami FL, USA).

\section{Conditioning of mesenchymal stem cell culture system with 5'azacitydyne}

MSCs basic culture medium was supplemented with $10 \mu \mathrm{mol} / /$ 5'azacitydyne (Sigma, Germany). MSCs were pre-incubated in medium with 5'azacitydyne for 24 hours. After that time, the medium was removed and MSCs were subcultivated for 14 days in basic medium.

\section{Conditioning of mesenchymal stem cell culture system with growth factors released from cardiomyocyte cell line}

Mesenchymal stem cells from bone marrow were clonally enriched. After 14 days of cultivation, cells were trypsinized with $0.25 \%$ Trypsin-EDTA (PAA, Austria). Clonal cells were seeded at a density of $5 \times 10^{3}$ cells onto $18 \mathrm{~mm}$ cover glasses (Witko, Poland) and placed into twelve-well culture plates. After 24 hours of pre-incubation, the basic medium was removed and mesenchymal stem cells were subcultivated for another 14 days in conditioned media obtained from the immortalized cardiomyocyte cell line.

\section{Fluorescence immunostaining as a detection tool for transdifferentiation}

For immunolabeling of cell sheets, clonal cells were plated onto cover glasses (Witko, Poland) and cultivated under various environmental conditions, such as 5'azacitydyne and conditioned medium for 14 days. After cultivation time, cells were fixed with $4 \%$ paraformaldehyde in PBS $(\mathrm{pH} 7.4)$ at room temperature for $20 \mathrm{~min}$., washed with PBS (3x5 min) and blocked with $0.1 \mathrm{M}$ glycine for $10 \mathrm{~min}$. Permeabilization was performed by incubation with $0.25 \%$ Triton X-100 in PBS for $10 \mathrm{~min}$. After incubation with $1 \%$ bovine serum albumin (BSA, Sigma, Germany) for $30 \mathrm{~min}$, cells were stained with rat-specific primary antibodies against calponin, $\alpha$-actin, sarcomeric actin, myogenin (1:100, Abcam, UK) and desmin (1:20, Abcam, UK) for $1 \mathrm{~h}$ at room temperature. Antibody binding was detected by TRITC-conjugated secondary antibodies (1:85, Sigma, Germany). Cell nuclei were labelled with DAPI (4',6'-diamino-2-phenylindole, Sigma, Germany). Finally, coverslips were rinsed three times with PBS for $5 \mathrm{~min}$ and 
mounted in Aqua-Poly/Mount (Polysciences, Germany) prior to observation by using Nikon C1 laser-scanning microscope (Nikon, Japan). In negative control experiments, 1\% PBS-BSA or equimolar concentrations of an irrelevant primary antibody replaced the primary antibody. Slides were examined by two independent observers and then images were captured using EZ-C1 software (Ver. 3.80; Nikon Instruments, USA). Specific markers expression was evaluated using the semiquantitative IRS scale (0-12 points), according to Remmele and Stegner, which considered both the intensity of the colour reaction (as marker of the level of antigen amount) and the percentage of positive cells. Terminal values from 1 to 2 indicate the low intensity of reaction, from 3 to 4 medium, while from 6 to 12 high intensity of the reaction. Each experiment was done in triplicate.

\section{Results}

Characteristic of bone marrow mesenchymal stem cells

Bone marrow mesenchymal stem cells propagated rapidly in vitro and constituted a homogenous fibroblast-like morphology (Fig. 1A). For phenotypic characterization, mesenchymal stem cells were assessed for the expression of CD90, CD34 and CD44. The putative stem cell markers CD90 and CD44 which have been previously reported to be expressed in the rat bone marrow mesenchymal stem cells at high-level Karaoz et al. [8], were confirmed to be strongly expressed (Fig.1B). Staining for CD34 was negative, only single cells were CD34+ (Fig.1B), which indicates the mesenchymal nature of isolated cells. The colony forming efficiency (CFE), which is considered to correlate with the number of stem cells, was calculated to be $0.22 \%$. Rat mesenchymal stem cells formed on average $11 \pm 2$ colonies (Fig. 1D). Clonal cells could be serially cultivated up to 4 passages before they revealed signs of senescence, the activity of beta-galactosidase (Fig. 1C). Additionally, adipogenic and osteogenic differentiation of MSCs was performed to confirm the multipotential properties of isolated bone marrow MSCs (Fig. 1E-F).

\section{Effect of environmental conditions on the}

differentiation of bone marrow mesenchymal stem cells

The effect of 5'azacitydyne and cardiomyocyte cell line conditioned medium on calponin, $\alpha$-actin, desmin, sarcomeric actin and myogenin expression was analyzed by immunofluorescence of clonal cells subcultivated in cover glasses. As expected, all muscle-specific markers appeared in mesenchymal stem cells due to the process of differentiation, but proportion of immunopositive cells showed significant differences depending on condition used. Conditioned medium from cardiomyocyte cell line ( $\mathrm{H} 9 \mathrm{C} 2)$, as well as, 5 'azacitydyne supplemented medium induced muscle differentiation process in bone marrow mesenchymal stem cells in comparison to the control (mesenchymal stem cells cultured alone) (Fig. 2A). Expression of desmin, $\alpha$-actin, sarcomeric actin and myogenin significantly increased after 14 days of bone marrow mesenchymal stem cells cultivation in 5'azacitydyne and $\mathrm{H} 9 \mathrm{C} 2$ conditioned media $(8,12,3,2$ and 4, 12, 4, 6 points, respectively, in IRS scale) (Fig. 2B-C).

\section{Discussion}

The injured human myocardium possesses a limited regenerative potential. Following acute myocardial infarction, tissue repair is deficient and irreplaceable loss of cardiac muscle overloads the surviving myocardium resulting in heart failure or death [9]. Despite many breakthroughs in cardiovascular medicine, current therapies for heart failure are of limited benefit in regaining lost cardiac function. Recently, cell therapy has emerged as an attractive therapeutic modality to repair damaged myocardium. Bone marrow mesenchymal stem cells are attractive for cell therapy, as they can give rise to various cell types, including cardiomyocytes, endothelial cells, and smooth muscle cells [10-12]. However, it is impossible to predict the differentiation pathway of implanted undifferentiated cells [13]. To reduce all side effects, the in vitro initiation of MSCs differentiation into cardiomyocytes can be performed but it can change stem cell properties. Moreover, it is well documented that implantation of differentiated into cardiomyocytes MSCs improves the function and regeneration of heart [14-16]. The possibility of the influence of neighbouring cardiomyocytes on MSCs differentiation is very interesting issue, as well.

Stem cells incubated with 5'azacitydyne changed their phenotype toward cardiomyocytes [17-19]. Tomita and coworkers [20] stated that $10 \mu \mathrm{M}$ 5'azacitydyne, added for 24 hours to in vitro culture, induced differentiation of mesenchymal stem cells. They also observed the creation of myotubule-like structures after 4 weeks from differentiation initiating. In our study in the same concentration of 5'azacitydyne we observed a strong expression of $\alpha$-actin and desmin, which are the early differentiation markers, and weaker expression of sarcomeric actin and myogenin. The gained results can be considered as reliable in comparison to results $\mathrm{Xu}$ et al. [21]. We did not examine the potential of cells to spontaneous contraction. Nevertheless, we cannot exclude the differentiation of MSCs towards cardiomyocytes phenotype. Data concerning the differentiation 


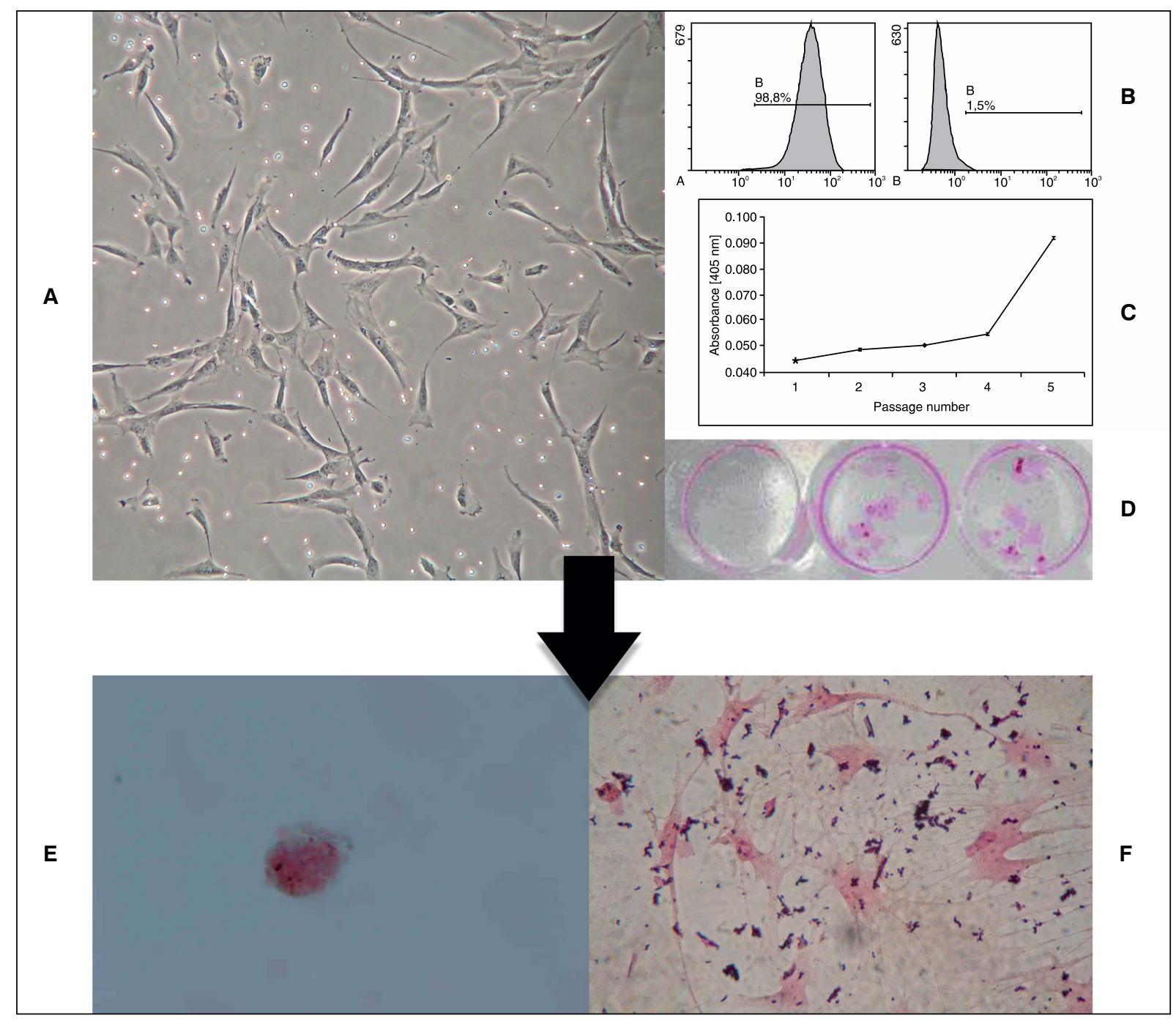

Figure 1. Evaluation of rat mesenchymal stem cells isolated from bone marrow: morphology in vitro (A), expression of $\mathrm{CD} 90$ and CD34 (B), aging process assay; $\beta$-galactosidase activity detection (C), clonogenity assay; rhodamine $\mathrm{B}$ staining indicates good visible red colonies $(D)$, adipogenic assay; oil red staining $(E)$, osteogenic assay; von Kossa test $(F)$

of stem cells by 5'azacitydyne influence are conflicting. Liu and coworkers [22] did not observe differentiation of rat bone marrow mesenchymal stem cells in cardiomyocytes with use of 3,5 and $10 \mu \mathrm{M}$ of 5'azacitydyne while $\mathrm{Xu}$ and coworkers had done [21]. The most probably, additional factors are required for successful cardiomyocytes differentiation process.

Experimental settings with the use of 5'azacitydyne are totally artificial. Such conditions do not reflect any aspect of the environment within native heart muscle tissue. That is why in our study we tried to find agents that control differentiation process towards cardiomyocyte lineage in the environment mimicking in vivo condition. We checked how conditioned medium influences on MSCs differentiation. After 14 days of culture in medium obtained from immortalized cardiomyocytes cell line, the transdifferentiation process was observed.
Morphology of MSCs has been changed into muscle phenotype. Expression of specific muscle markers such as desmin, $\alpha$-actin, sarcomeric actin and myogenin appeared following incubation with conditioned media. All observations support the evidences for the induction of transdifferentiation toward muscle phenotype. Expression of sarcomeric actin and myogenin was at medium and high level (4 and 6 points, respectively, in IRS scale). These results are in opposite to Wang and coworkers [23] who stated that cell fusion was responsible for differentiation. We proved that conditioned medium has an influence on changes of MSCs phenotype. We think that the differentiation process is induced by signals from cardiomyocytes, which release paracrine factors.

It was noticed that the ageing process could be visible after a few passages of MSCs. The aim of 


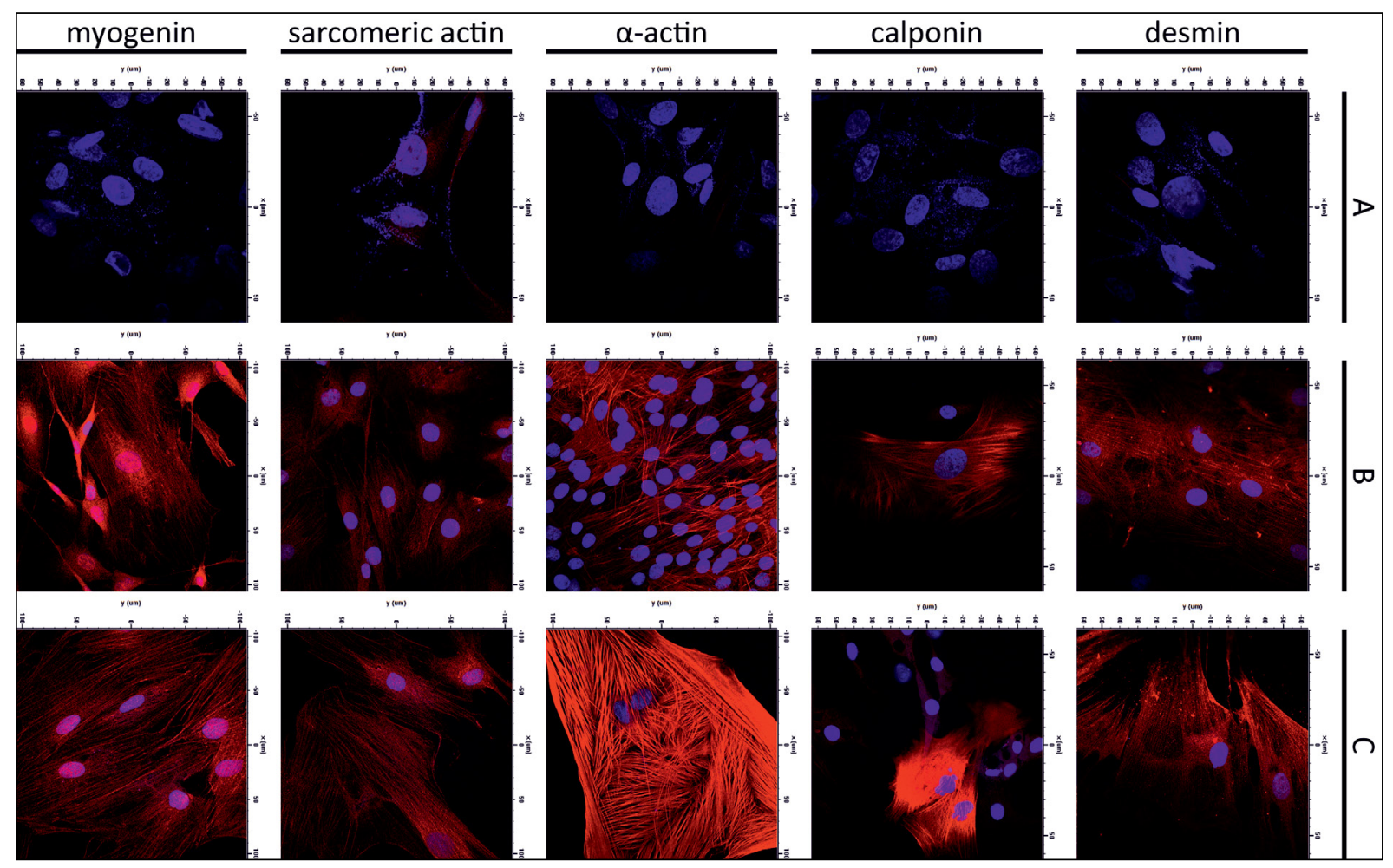

Figure 2. Immunofluorescence staining for desmin, calponin, $\alpha$-actin, sarcomeric actin, myogenin in bone marrow mesenchymal stem cells (A), MSC cultured in conditioned medium supplemented by 5'azacitydyne (B), MSC cultured in conditioned medium collected from $\mathrm{H} 9 \mathrm{C} 2$ cell line $(\mathrm{C})$. Bar $=50 \mu \mathrm{m}$. Nuclei were stained with DAPI

transplanted stem cells is not only temporary muscle tissue regeneration but also replenishment of stem cell population to sustain the effect of treatment. The important question focuses on the ageing of cells used in the long-term effect of therapy. It is also still not known whether a single or multiply injections would be sufficient to bring a stable functional improvement over a long time due to homing mechanisms are not well defined [24-28]. Another issue that needs to be considered is the number of cells delivered at the site of engraftment and the prevention of possible ageing process of injected cells [25].

\section{Conclusions}

Stem cells therapy of myocardium infarction offers the possibility to reconstruct the heart tissue. Our study shows that conditioned medium provides the inductive signals to initiate transdifferentiation of bone marrow mesenchymal stem cells cardiomyocytes-like phenotype. The presented data support the conclusion that this therapeutic concept may represent a promising strategy for the repair and reconstruction of the heart tissue, however performing further experiments with co-culture of MSCs and cardiomyocytes are necessary.

\section{References}

1. Joggerst SJ, Hatzopoulos AK. Stem cell therapy for cardiac repair: benefits and barriers. Expert Rev Mol Med. 2009; 11: e20, doi: 10.1017/S1462399409001124, indexed in Pubmed: 19586557.

2. Nguyen BK, Maltais S, Perrault LP, et al. Improved function and myocardial repair of infarcted heart by intracoronary injection of mesenchymal stem cell-derived growth factors. J Cardiovasc Transl Res. 2010; 3(5): 547-558, doi: 10.1007/s12265-010-9171-0, indexed in Pubmed: 20559784.

3. Shabbir A, Zisa D, Suzuki G, et al. Heart failure therapy mediated by the trophic activities of bone marrow mesenchymal stem cells: a noninvasive therapeutic regimen. Am J Physiol Heart Circ Physiol. 2009; 296(6): H1888-H1897, doi: 10.1152/ajpheart.00186.2009, indexed in Pubmed: 19395555

4. He XQ, Chen MS, Li SH, et al. Co-culture with cardiomyocytes enhanced the myogenic conversion of mesenchymal stromal cells in a dose-dependent manner. Mol Cell Biochem. 2010; 339(1-2): 89-98, doi: 10.1007/s11010-009-0372-2, indexed in Pubmed: 20063193.

5. Pittenger M. Sleuthing the source of regeneration by MSCs. Cell Stem Cell. 2009; 5(1): 8-10, doi: 10.1016/j.stem.2009.06.013, indexed in Pubmed: 19570508

6. Furuta A, Carr LK, Yoshimura N, et al. Advances in the understanding of sress urinary incontinence and the promise of stem-cell therapy. Rev Urol. 2007; 9(3): 106-112, indexed in Pubmed: 17934567.

7. Lennon DP, Caplan Al. Isolation of rat marrow-derived mesenchymal stem cells. Exp Hematol. 2006; 34(11): 1606-1607, doi: 10.1016/j. exphem.2006.07.015, indexed in Pubmed: 17046584

8. Karaoz E, Aksoy A, Ayhan S, et al. Characterization of mesenchymal stem cells from rat bone marrow: ultrastructural properties, differentiation potential and immunophenotypic markers. Histochem Cell Biol. 2009; 132(5): 533-546, doi: 10.1007/s00418-009-0629-6, indexed in Pubmed: 19688349.

9. Roura S, Farré J, Hove-Madsen L, et al. Exposure to cardiomyogenic stimuli fails to transdifferentiate human umbilical cord blood-derived mesenchymal stem cells. Basic Res Cardiol. 2010; 
105(3): 419-430, doi: 10.1007/s00395-009-0081-8, indexed in Pubmed: 20091315

10. Carvalho KA, Oliveira L, Malvezzi M, et al. Immunophenotypic expres sion by flow cytometric analysis of cocultured skeletal muscle and bone marrow mesenchymal stem cells for therapy into myocardium. Transplant Proc. 2008; 40(3): 842-844, doi: 10.1016/j.transproceed.2008.02.053, indexed in Pubmed: 18455032.

11. Gallo MP, Ramella R, Alloatti G, et al. Limited plasticity of mesenchyma stem cells cocultured with adult cardiomyocytes. J Cell Biochem. 2007; 100(1): 86-99, doi: 10.1002/jcb.21012, indexed in Pubmed 16888800.

12. Kim H, Kim SW, Nam D, et al. Cell therapy with bone marrow cells for myocardial regeneration. Antioxid Redox Signal. 2009; 11(8): 1897 1911, doi: 10.1089/ars.2009.2486, indexed in Pubmed: 19203213.

13. Li X, Yu X, Lin Q, et al. Bone marrow mesenchymal stem cells differentiate into functional cardiac phenotypes by cardiac microenvironment. J Mol Cell Cardiol. 2007; 42(2): 295-303, doi: 10.1016/j. yimcc.2006.07.002, indexed in Pubmed: 16919679.

14. Antonitsis P, loannidou-Papagiannaki E, Kaidoglou A, et al. In vitro cardiomyogenic differentiation of adult human bone marrow mesenchymal stem cells. The role of 5-azacytidine. Interact Cardiovasc Thorac Surg. 2007; 6(5): 593-597, doi: 10.1510/icvts.2007.157875, indexed in Pubmed: 17670726.

15. Gwak SJ, Bhang SH, Yang HS, et al. In vitro cardiomyogenic differentiation of adipose-derived stromal cells using transforming growth factor-beta1. Cell Biochem Funct. 2009; 27(3): 148-154, doi: 10.1002/cbf.1547, indexed in Pubmed: 19319827.

16. Shim WSN, Jiang $S$, Wong $P$, et al. Ex vivo differentiation of human adult bone marrow stem cells into cardiomyocyte-like cells. Biochem Biophys Res Commun. 2004; 324(2): 481-488, doi: 10.1016/j. bbrc.2004.09.087, indexed in Pubmed: 15474453.

17. Balana B, Nicoletti C, Zahanich I, et al. 5-Azacytidine induces changes in electrophysiological properties of human mesenchymal stem cells. Cell Res. 2006; 16(12): 949-960, doi: 10.1038/sj.cr.7310116, indexed in Pubmed: 17160070.

18. Corti S, Strazzer S, Del Bo R, et al. A subpopulation of murine bone marrow cells fully differentiates along the myogenic pathway and participates in muscle repair in the mdx dystrophic mouse. Exp Cell
Res. 2002; 277(1): 74-85, doi: 10.1006/excr.2002.5543, indexed in Pubmed: 12061818

19. Dai W, Hale SL, Kloner RA. Stem cell transplantation for the treatment of myocardial infarction. Transpl Immunol. 2005; 15(2): 91-97, doi: 10.1016/j.trim.2005.09.004, indexed in Pubmed: 16412954

20. Tomita S, Li RK, Weisel RD, et al. Autologous transplantation of bone marrow cells improves damaged heart function. Circulation. 1999; 100(19 Suppl): |l247-||256, indexed in Pubmed: 10567312.

21. Xu W, Zhang X, Qian H, et al. Mesenchymal stem cells from adult human bone marrow differentiate into a cardiomyocyte phenotype in vitro. Exp Biol Med (Maywood). 2004; 229(7): 623-631, indexed in Pubmed: 15229356

22. Liu Yu, Song J, Liu W, et al. Growth and differentiation of rat bone marrow stromal cells: does 5-azacytidine trigger their cardiomyogenic differentiation? Cardiovasc Res. 2003; 58(2): 460-468, indexed in Pubmed: 12757880

23. Wang $T, X u Z$, Jiang $W$, et al. Cell-to-cell contact induces mesenchymal stem cell to differentiate into cardiomyocyte and smooth muscle cell. Int J Cardiol. 2006; 109(1): 74-81, doi: 10.1016/j.ijcard.2005.05.072, indexed in Pubmed: 16122823

24. Baharvand $\mathrm{H}$, Azarnia $\mathrm{M}$, Parivar $\mathrm{K}$, et al. The effect of extracellular matrix on embryonic stem cell-derived cardiomyocytes. J Mol Cell Cardiol. 2005; 38(3): 495-503, doi: 10.1016/j.yjmcc.2004.12.011, indexed in Pubmed: 15733909.

25. Bongso A, Fong CY, Gauthaman K. Taking stem cells to the clinic: Major challenges. J Cell Biochem. 2008; 105(6): 1352-1360, doi: 10.1002/jcb.21957, indexed in Pubmed: 18980213.

26. Choumerianou DM, Dimitriou H, Kalmanti M. Stem cells: promises versus limitations. Tissue Eng Part B Rev. 2008; 14(1): 53-60, doi: 10.1089/teb.2007.0216, indexed in Pubmed: 18454634.

27. Hilenski LL, Terracio L, Borg TK. Myofibrillar and cytoskeletal assembly in neonatal rat cardiac myocytes cultured on laminin and collagen. Cell Tissue Res. 1991; 264(3): 577-587, indexed in Pubmed: 1907887.

28. van Dijk A, Niessen HWM, Ursem W, et al. Accumulation of fibronectin in the heart after myocardial infarction: a putative stimulator of adhesion and proliferation of adipose-derived stem cells. Cell Tissue Res. 2008; 332(2): 289-298, doi: 10.1007/s00441-008-0573-0, indexed in Pubmed: 18305959. 